\section{$\underset{\substack{\text { hommes } \\ \text { \& migrations }}}{ }$}

\section{Hommes \& migrations}

Revue française de référence sur les dynamiques

migratoires

$1293 \mid 2011$

L'immigration dans les musées

\title{
Esclavage, colonialisme et représentations muséales en Grande-Bretagne
}

Anciens et nouveaux circuits de migration

\section{Stephen Small}

Traducteur : Françoise Bouillot

\section{(2) OpenEdition Journals}

\section{Édition électronique}

URL : http://journals.openedition.org/hommesmigrations/507

DOI : 10.4000/hommesmigrations.507

ISSN : 2262-3353

Éditeur

Musée national de l'histoire de l'immigration

Édition imprimée

Date de publication : 1 septembre 2011

Pagination : 74-84

ISSN : 1142-852X

Référence électronique

Stephen Small, «Esclavage, colonialisme et représentations muséales en Grande-Bretagne », Hommes \& migrations [En ligne], 1293 | 2011, mis en ligne le 31 décembre 2013, consulté le 19 avril 2019. URL : http://journals.openedition.org/hommesmigrations/507; DOI : 10.4000/hommesmigrations.507 


\section{Esclavage, colonialisme et représentations muséales en Grande-Bretagne} Anciens et nouveaux circuits de migration

Par Stephen Small, professeur associé, African American Studies, université de Californie, commissaire invité et membre du conseil scientifique de l'Atlantic Slave Trade Gallery de Liverpool

Historiquement, la plupart des musées en Grande-Bretagne ont eu pour fonction de soutenir la fierté nationale et de glorifier la culture britannique, en exposant toute une gamme d'objets amassés au cours de la période coloniale. L'accroissement des populations d'origine immigrée sur le territoire britannique remet en cause ces pratiques muséales. Un chercheur engagé dans le débat sur les réparations symboliques décrypte les rapports complexes entre les anciens migrants et les musées. 
En Grande-Bretagne, dans les années cinquante, la population noire était peu nombreuse et concentrée dans les villes portuaires historiques de Liverpool, Londres et Cardiff ${ }^{11}$. En 2011, cette même population compte plus de 1,5 million de personnes, réparties dans toutes les grandes villes. Il existe par ailleurs une communauté plus nombreuse encore d'immigrés issus de l'Inde, du Pakistan et du Bangladesh : en 2011, ils étaient plus de 3 millions. En outre, au cours de ces dix dernières années, la GrandeBretagne a vu l'arrivée de milliers de nouveaux migrants issus de toute l'Europe, ainsi que des réfugiés de nations africaines qui n'appartenaient pas à l'Empire britannique (comme la Somalie, le Congo et le Rwanda).

Dans les années cinquante, la plupart des musées britanniques ignoraient ou marginalisaient la question de l'esclavage et de son héritage. Quand ils abordaient ces questions, c'était pour glorifier l'Empire ou l'abolition par les Britanniques du commerce des esclaves, et ils s'intéressaient davantage à la culture matérielle qu'aux hommes. Les musées exposaient des objets et des tableaux présentant des stéréotypes grossiers et unidimensionnels de l'Afrique, des Africains et de l'esclavage ; en outre, ils n'avaient quasiment aucun Noir parmi leurs agents ou leurs gestionnaires. Aujourd'hui, en revanche, il semble que l'esclavage et son héritage soient devenus un aspect clé des musées britanniques qui ont organisé des expositions temporaires ou permanentes $^{(2)}$ sur le sujet. Certains ont désormais des expositions permanentes et des galeries consacrées à l'esclavage, au colonialisme ou à l'Afrique. Celles-ci sont en général bien documentées, voire progressistes, et s'efforcent de rendre compte de la diversité de l'expérience noire, en Afrique comme dans la diaspora. Plusieurs témoignent d'une critique significative de l'esclavage, de l'abolition du commerce triangulaire et même de l'Empire. Les Noirs sont plus activement impliqués qu'ils ne l'ont jamais été - en tant que curateurs, directeurs ou rédacteurs -, bien que dans une proportion qui reste faible par rapport à leur population totale $\mathrm{e}^{(3)}$. Il y a eu en outre un gros travail de sensibilisation pour favoriser les ressources et les informations issues de la diaspora africaine et de l'Afrique elle-même. Certaines de ces activités ont été lancées voici plusieurs décennies, avec pour perspective et point culminant l'année 2007 , date du $200^{\circ}$ anniversaire de l'abolition légale du commerce des esclaves dans l'Empire britannique. Cet anniversaire a suscité de nombreuses recherches et une grande diversité d'initiatives muséales qui ont bénéficié d'un financement conséquent de la part du gouvernement. Beaucoup d'organisations noires et multiraciales se sont impliquées dans ces initiatives, et les demandes de réparation se sont multipliées ${ }^{(4)}$.

La migration et les musées mettent en lumière deux domaines qui se chevauchent tout en étant inextricablement liés. En quoi les musées sont-ils importants pour penser les migrations? Les musées sont des sites cruciaux de contestation de l'identité 
et de l'ethnicité, y compris de l'identité nationale et religieuse ${ }^{(5)}$. Ils sont aussi d'importants sites de contestation des grandes narrations historiques, en particulier de l'histoire nationaliste et impériale ${ }^{(6)}$. Mais parmi les sites institutionnels, certains continuent d'énoncer des discours hostiles aux Noirs et à l'Afrique ${ }^{(\gamma)}$. Dans tous les cas, les musées soulèvent des questions de pouvoir, d'inégalité et d'accès aux ressources ${ }^{(8)}$. Il est frappant de voir que les transformations les plus décisives des expositions en Grande-Bretagne au cours des cinquante dernières années sont directement liées à l'apparition d'une immigration à grande échelle.

\section{Un chevauchement de quatre migrations}

Quatre types de migrations mettent en lumière les liens entre les musées et la migration, chacune continuant à être modelée par la classe sociale, la race et le genre. Tout d'abord, la migration des Européens en direction de l'Afrique, de l'Asie et de ce que l'on appelle aujourd'hui les Amériques ${ }^{(9)}$. Sans ces migrations et les implantations de colonies qu'a entraînées la conquête au cours des cinq cents dernières années, de nombreux musées de Grande-Bretagne seraient radicalement différents, voire quasiment vides. Pendant plusieurs centaines d'années, ces musées ont servi à célébrer l'Empire, à promouvoir la gloire impériale, à soutenir la fierté nationale (blanche) et à glorifier la culture occidentale en exhibant une vaste gamme d'objets pillés et amassés à l'ère de l'esclavage et du colonialisme européens. La question du genre a également été centrale dans la gestion et les représentations de ces musées.

En second lieu, la migration massive d'Antillais et d'Afro-Caribéens vers la GrandeBretagne à partir des années cinquante $\mathrm{a}$ fait exploser la démographie des populations noires de villes portuaires comme Liverpool, Londres et Cardiff. À tel point qu'on est passé d'une population de moins de 50000 personnes dans les années cinquante à une population de plus de 1,5 million de personnes en $2011^{(10)}$.

En troisième lieu, la migration d'autres groupes issus de l'Empire britannique, notamment des Asiatiques venus de l'Inde, du Pakistan, du Bangladesh et de l'Afrique de l'Est ${ }^{(11)}$, fait de cette population la plus importante communauté qui rassemble plus de 3 millions de personnes.

Enfin, on a vu se développer depuis les années quatre-vingt des modèles de migrations et d'implantation radicalement nouveaux, d'une grande importance démographique et politiquement significatifs. Ils concernent la migration à grande échelle de Blancs issus de toute l'Europe, et surtout de l'ancienne Europe de l'Est. Il faut compter en outre des dizaines de milliers de réfugiés et de demandeurs d'asile venus essentiel- 
lement d'Afrique et d'Asie. Si leur implication dans les musées reste pour l'instant négligeable, il est probable qu'à mesure qu'ils affermiront leur installation, ils réclameront à leur tour des représentations et des expositions prenant en considération leurs histoires particulières.

Toutes ces migrations d'hommes et de femmes étaient et demeurent fortement genrées, reflétant les divisions prévalentes du travail et l'affectation des hommes et des femmes à des domaines différents de la production et de la reproduction à chaque stade de la migration. La migration des Européens au cours de la période de l'esclavage et du colonialisme a impliqué bien plus d'hommes blancs que de femmes. Le commerce européen des esclaves a concerné bien plus d'hommes africains que de femmes, parce que les planteurs préféraient acheter des hommes. Et la proportion de femmes blanches s'est considérablement accrue aux États-Unis, alors que ce ne fut pas le cas dans les Caraïbes ${ }^{(12)}$. La migration des Antillais vers la Grande-Bretagne a impliqué une majorité d'hommes, mais avec un nombre significatif de femmes. $\mathrm{Si}$

\section{Les femmes noires ont été actives dans toutes les initiatives visant à transformer} les représentations muséales au cours de ces vingt dernières années, et restent au premier plan des mouvements actuels, y compris sur la question des réparations. la plupart sont venues en tant qu'épouses ou filles de migrants, beaucoup d'autres sont arrivées seules ${ }^{(13)}$, reflet de l'indépendance économique de nombre de femmes noires dans les Caraibes britanniques. Parmi les migrants asiatiques, les hommes étaient très nettement surreprésentés, les femmes arrivant essentiellement en tant qu'épouses ou membres de la famille. Les formes de migrations ont beau avoir considérablement changé au fil du temps, elles continuent à concerner une plus forte proportion d'hommes que de femmes.

La question du genre a eu sur les musées un impact significatif. Les plus importants musées britanniques du XIX ${ }^{\mathrm{e}}$ siècle témoignent d'une fascination pour la masculinité telle qu'elle apparaît dans le combat militaire, fascination qui a perduré dans le développement muséal ultérieur. Les stéréotypes sexistes de la femme ont prévalu depuis la naissance des musées - ceux des femmes noires étant les plus dégradants. Les femmes ont été moins impliquées que les hommes dans la gestion des musées, alors même que les femmes noires étaient plus actives que les hommes dans les petites organisations communautaires chargées des commémorations et de la mémoire de l'esclavage ${ }^{(14)}$. Les femmes noires ont été actives dans toutes les initiatives visant à transformer les représentations muséales au cours de ces vingt dernières années, et restent au premier plan des mouvements actuels, y compris sur la question des réparations. 


\section{Musées et Empire en Grande-Bretagne}

Dans les années qui ont suivi la Seconde Guerre mondiale, les musées n'entraient pas dans les préoccupations de la majorité des Noirs dont les priorités étaient l'emploi et le logement, auxquels se sont ajoutés au fil du temps l'éducation des enfants et l'accès à la politique. À toutes les époques ils durent affronter le racisme, qui les affectait directement dans tous ces domaines, et dénoncer les images hostiles et racistes à la télévision, dans la presse et d'autres médias. Mais il reste que la majorité des Noirs ne s'intéressait pas aux musées ${ }^{(15)}$.

Certes, des problèmes de commémoration se posaient dans les communautés noires, au sein des églises et des organisations communautaires. Ces groupes avaient des objets, des monuments, des mémoriaux, mais qui restaient à une échelle personnelle, sur une base familiale ou communautaire. Les organisations rastafariennes ont joué ici un rôle central, surtout pour la deuxième génération - des gens comme moi, nés de parents immigrés des Caraïbes ${ }^{(16)}$.

Les rastafariens apportaient des images, des objets artisanaux et sacrés de toute l'Afrique - d'Éthiopie en particulier -, et aussi des idées et des icônes de la Jamaïque et des Caraïbes ${ }^{(17)}$. Mais là encore, ces groupes n'avaient aucun lien avec les musées, disposant de leurs propres arrangements institutionnels pour la commémoration et la mémorialisation. Les années quatrevingt furent marquées par une frustration croissante des communautés noires vis-à-vis des représentations stéréoty-

pées des Noirs dans les musées. C'est alors que certaines initiatives émergèrent 
pour y remédier ${ }^{(18)}$. Ce fut le député Bernie Grant qui plaça les musées sur l'agenda de la communauté noire. Bernie Grant était l'un des trois politiciens noirs élus au Parlement en 1987, avec Diane Abbott et Paul Boateng. Tous les trois étaient membres du Parti travailliste et représentaient des circonscriptions londoniennes. Grant était né en Guyane et avait émigré très jeune en Grande-Bretagne où il fut un syndicaliste actif avant de devenir le leader du Conseil londonien pour l'égalité raciale de Haringey, et d'être élu député de Tottenham - un district ouvrier multiracial du nord de Londres. Il fut en 1989 un membre fondateur du Parliamentary Black Caucus, modelé sur le Congressional Black Caucus des États-Unis. Tout son travail témoignait de sa priorité, qui était de venir en aide aux ouvriers, qu'ils soient noirs ou blancs. Il était activement engagé dans la lutte contre le racisme et les inégalités en Grande-Bretagne, et dans la question de l'héritage de l'esclavage et du colonialisme dans les Caraïbes et en Afrique. Il s'intéressa aux communautés noires de Grande-Bretagne et d'Europe, et s'impliqua dans les organisations et les conférences panafricaines. En outre, il initia et mena le plus grand mouvement britannique de réparations pour l'esclavage.

\section{Demandes de réparation et de restitution des objets}

Bernie Grant savait que les Britanniques avaient volé, emprunté ou extorqué des milliers d'objets dans tout l'Empire et les avaient exposés partout dans le pays ou enfermés dans les caves de leurs musées. Il organisa une protestation au British Museum, appelant à rendre ces objets sacrés à leurs propriétaires légitimes, et lança le Mouvement des réparations qui mobilisa des milliers de personnes. Il identifia les bronzes du Bénin comme l'un des plus importants ensembles d'objets pillés par les Britanniques - des centaines de sculptures volées en $1897^{(19)}$. Un épisode historique bien connu, le meurtre d'un Blanc sur la terre de l'oba du Bénin ${ }^{(20)}$ devint le prétexte à une expédition punitive qui entraîna la destruction de Benin City et le pillage des bronzes. Ceux-ci, actuellement estimés à plusieurs millions de dollars, sont en partie conservés au British Museum, au musée de Liverpool et dispersés un peu partout ailleurs en Europe. Ils sont l'emblème de cet entrelacs de politique, de profit et de culture qui, associé à une arrogance nationale et raciste, reste l'un des legs directs de l'esclavage et du colonialisme. Le retour des bronzes est une question qui reste en suspens, et il ne fait pas de doute que bien d'autres éléments dissimulés dans tous les musées de ce pays attendent encore d'être inventoriés.

Au cours d'une visite du Centre communautaire Charles Wootton de Liverpool - 
ainsi nommé en mémoire d'un Noir assassiné par des Blancs en 1919 -, Bernie Grant m'a recruté pour travailler avec lui. Je travaillais alors à l'Atlantic Slave Trade Gallery de Liverpool, et je m'intéressais à son activisme et à sa façon d'associer les musées et les réparations à d'autres priorités de la communauté noire, comme l'emploi, l'éducation et le maintien de l'ordre. Je savais que le musée de Liverpool possédait plusieurs bronzes du Bénin. Beaucoup de Noirs de Liverpool avaient fait campagne pour un musée noir, convaincus que les musées possédaient des milliers d'autres objets. Ils avaient aussi protesté contre le racisme dans les musées de Liverpool ${ }^{(21)}$. Je m'intéressais beaucoup à la problématique des réparations - à cause des questions qu'elle soulevait sur les profits de l'esclavage, et parce qu'elle offrait un fort potentiel de mobilisation des Noirs et de leurs alliés blancs sur le legs de l'esclavage et de l'éducation. Bernie Grant s'impliqua dans l'Atlantic Slave Trade Gallery alors en cours de constitution à Liverpool, et j'écrivis un chapitre sur le legs de l'esclavage dans le catalogue de la galerie ${ }^{(22)}$.

Au cours de la décennie suivante, je suis devenu l'assistant de Bernie Grant, chargé notamment des recherches sur les musées. Entre 1992 et 1995,j'ai donné au nom de son organisation - Reparations UK - des conférences dans de nombreuses universités de Grande-Bretagne. Je l'ai encouragé à placer les musées au cceur du débat sur les réparations - puisqu'ils possèdent des éléments concrets collectés durant l'esclavage et le colonialisme que nous pouvons identifier et réclamer. Pour nous, il ne s'agissait pas simplement de changer les images et les objets, mais bien de mobiliser les populations sur l'accès aux ressources, parmi lesquelles l'éducation et l'emploi. Nous avons soulevé des questions sur le nombre d'hommes et de femmes noirs employés dans les musées, sur les représentations de l'Afrique, sur les nombreux mensonges sur l'esclavage, et nous avons remis en cause la prétendue magnanimité de l'abolition légale du commerce des esclaves par les Britanniques. Ce fut pour toutes ces raisons que ce mouvement reçut autant de soutiens. Bernie Grant mourut prématurément du diabète en 2000, à l'âge de 57 ans. Le Mouvement des réparations se poursuit et s'élargit, mais les musées ne sont plus une préoccupation centrale.

\section{D'une vision édulcorée de l'histoire à la concurrence des mémoires}

Nous devons nous interroger sur la façon dont la permanence des inégalités dans l'accès aux ressources et au pouvoir va affecter les représentations de l'esclavage, du colonialisme et de leur héritage. Tout d'abord, quelle est et quelle sera la nature des histoires que racontent les musées? Il faudra veiller à empêcher les narrations et les 
représentations de devenir monotones, anodines et vides de toute discussion du conflit situé au cceur de l'esclavage. Je redoute une dérive vers des narrations effaçant la violence, la brutalité et l'exploitation extrêmes du colonialisme. Nous avons vu ce modèle s'installer à travers le sud des États-Unis, où les musées qui célébraient et glorifiaient autrefois l'esclavage se contentent aujourd'hui de mentionner de façon simpliste la population noire. J'ai décrit ce phénomène comme une annihilation symbolique dans mes travaux sur les ÉtatsUnis $^{(23)}$. Il reste des signes prometteurs d'une résistance à un tel processus - grâce à des organisations noires et multiraciales comme le Centre Franz Fanon de Birmingham, le

\section{Beaucoup de Noirs de Liverpool avaient fait campagne pour un musée noir, convaincus que les musées possédaient des milliers d'autres objets.}

Centre Kuumba Imani Millenium de Liverpool ou le George Patmore Institute de Londres. En outre, le dynamique Mouvement des réparations peut continuer à soulever des questions sur la source et la légitimité des objets présentés dans les musées. Pour résister à de tels développements, je crois qu'un engagement continu avec l'Afrique et la diaspora des États-Unis et des Caraïbes devrait jouer un rôle clé, par le biais des échanges d'idées, des institutions, des idéologies et des ressources. Cela s'est toujours produit en Grande-Bretagne et se produit de plus en plus souvent dans d'autres nations d'Europe comme les Pays-Bas, l'Espagne et le Portugal ${ }^{(24)}$.

Un second point concerne le potentiel de réponses réactionnaires. Dans la GrandeBretagne actuelle, avec la fin du multiculturalisme, l'insistance accrue sur la cohésion sociale qui efface la discrimination raciale et l'inégalité, et les célébrations révisionnistes de l'Empire, l'héritage de l'esclavage pourrait se résumer à une simple célébration de l'abolitionnisme britannique. Le changement récent de gouvernement en faveur des conservateurs et des libéraux, l'aggravation des conditions économiques et les coupes budgétaires intensifient déjà la rivalité pour l'obtention de ressources qui va aussi affecter les musées.

Un troisième défi est celui qui peut venir de multiples groupes en compétition pour l'accès au musée et aux représentations. Les Noirs ne sont pas les seuls à vouloir que leur histoire soit racontée. Avec de multiples groupes de migrants issus de toutes les nations du monde, dont beaucoup sortent de situations de violence politique et de conflit armé, il y a bien des histoires à raconter, chacune étant considérée comme plus importante que les autres. De l'ancienne Yougoslavie à la Pologne, de la Somalie et du Congo au Rwanda, il y aura sans doute une rivalité accrue pour l'affirmation et l'exposition des mémoires. Certains groupes collaboreront, tandis que d'autres se feront concurrence. 


\section{Conclusion}

Après plus d'un siècle de musées britanniques conçus comme des bastions de la gloire impériale, on a assisté ces trente dernières années à une transformation des représentations et des discours sur le colonialisme. Des expositions, des galeries et même des musées ont été créés qui défient cette mémoire unidimensionnelle et déformée du passé. Des changements significatifs ont été obtenus ${ }^{(25)}$. Nous avons désormais un éventail de représentations plus vaste qu'il ne l'a jamais été. Ces transformations tiennent avant tout aux immigrants et à l'installation d'anciens sujets coloniaux dans la métropole ainsi qu'à leur mobilisation dans le cadre d'organisations multiraciales. Une partie des images les plus grossières et les plus offensantes présentées dans les musées ont été remises en cause. Plusieurs expositions ont été montées afin de présenter un point de vue plus équilibré sur l'esclavage et son héritage. Mais elles sont loin d'être exhaustives et leur statut reste précaire. En même temps, les demandes des nouveaux migrants ne cessent de croître. Au moment où la lutte contre les représentations historiquement dominantes du colonialisme dans les musées britanniques prend de l'ampleur, de nouvelles formes de migrations risquent de la remettre en cause. Il s'agit de savoir si ces mutations récentes vont freiner voire affaiblir les quelques gains obtenus, ou s'ils vont conduire à des transformations plus fondamentales dans les représentations muséales de l'immigration.

Parce que les musées sont des institutions racialisées, parce qu' ils continuent à abriter tant d'objets précieux et sacrés qui ont été volés ou acquis de façon illégitime, parce qu'ils sont une institution parmi d'autres de la contestation des grandes narrations de l'histoire nationale, parce que les musées sur les Noirs sont apparus avant tout du fait de multiples modèles de migrations, et parce qu' ils reflètent des questions d'accès aux ressources, de pouvoir et d'inégalité, le lien entre musées et migration reste une importante question pour les analystes sociaux. Les musées ne sont pas la question la plus importante qu'affronte la communauté noire en Grande-Bretagne. Les problèmes d'inégalité et de discrimination raciales et les conflits permanents avec la police restent bien plus fondamentaux. Les représentations étroites et déformées des Noirs que continuent si souvent à nous présenter les médias sont aussi très problématiques. Mais ce qui se passe dans les musées est significatif et requiert notre attention. Ils restent d'importantes institutions au vue des histoires qu'ils racontent sur l'esclavage, le colonialisme et la gloire impériale, et ils témoignent de la façon dont les migrations ne cessent de modeler les institutions britanniques. À cet égard, ce sont des institutions clés, qui offrent aux populations de Grande-Bretagne un accès aux représentations du passé. Nous devons donc continuer à nous montrer vigilants sur l'intrication entre les mouvements de migration et les musées.

Traduit de l'anglais par Françoise Bouillot 


\section{Bibliographie}

- Brennan Ferne, "Race, rights reparations : exploring a reparation framework for addressing trade inequality", in Hamline Journal of Public Law and Policy, vol. 30, $\mathrm{n}^{\circ}$ 1, automne 2008.

- Brennan Ferne, "Are reparations for slavery Justified?", in Human Rights Global Focus Group, Inde, International Human Rights Foundation, vol. 2, n 1, mars 2005, pp. 5-17.

- Bryan Beverley, Dadzie Stella et Scafe Suzanne, The Heart of the Race. Black Women's Lives in Britain, Londres, Virago, 1985.

- Campbell Horace, Rasta and Resistance. From Marcus Garvey to Walter Rodney, Londres, A Hansib Publication, 1985.

- Coombes Annie E., Reinventing Africa. Museums, Material Culture and Popular Imagination in Late Victorian and Edwardian England, New Haven et Londres, Yale University Press, 1994.

- Eichstedt Jennifer L. et Small Stephen, Representations of Slavery. Race, Ideology and Southern Plantations Museums, Washington DC, Smithsonian Institution Press, 2002.

- Fryer Peter, Staying Power : The History of Black People in Britain, Pluto Press, Londres, 1984.

- Gifford Lord, Brown Wally et Bundy Ruth, Loosen the Shackles. First Report of the Liverpool 8 Inquiry into Race Relations in Liverpool, Londres, Karia Press, 1989.

- Greenhill Eilean Hooper (dir.), Museums and Multiculturalism in Britain, Leicester, Leicester University Press, 1997.

- Gouldbourne Harry (dir.), Black Politics in Britain, Avebury, USA, Aldeshot and Brookfield, 1990.

- Hine Darlene Clark, Keaton Trica Danielle et Small Stephen, Black Europe and the African Diaspora, Urbana Champagne, University of Illinois Press, 2009.

- Littler Jo et Naidoo Roshi, (dir.), The Politics of Heritage. The Legacies of "Race", Routledge, Londres et New York, 2005.

- Miles Robert et Phizacklea Annie, White Man's Country : Racism in British Politics, Londres, Pluto Press, 1984.

- Morgan Jennifer L., Laboring Women : Reproduction and Gender in New World Slavery, Philadelphie, University of Pennsylvania Press, 2004.

- Nimako Kwame et Small Stephen, "The unfinished business of emancipation : the legacies of anglophone and dutch abolition", article présenté à l'Unfinished Emancipation and Black European Thinkers Symposium, National Institute for the Study of Dutch Slavery and its Legacy, Amsterdam, 29-30 juin 2009.

- Rose Eliot Joseph Benn et al., Colour and Citizenship : A Report on British Race Relations, Londres, Oxford University Press for Institute of Race Relations, 1969.

- Simpson Mooira G., Making Representations: Museums in the Post-Colonial Era, Londres et New York, Routledge, 2001 (1996).

- Sivanandan Ambalavaner, Communities of Resistance. Writings on Black Struggles for Socialism, Londres et New York, Verso, 1990.

- Small Stephen, "Contextualizing the Black presence in British Museums : representations, resources and response," in Greenhill Eileen Hooper (dir), Museums and Multiculturalism in Britain, Leicester, Leicester University Press, 1997, pp. 50-66.

- Small Stephen, Racialised Barriers. The Black Experience in the United States and England, New York et Londres, Routledge, 1994.

- Small Stephen, "The general legacy of the atlantic slave trade", in Tibbles Tony (dir.), Transatlantic Slavery. Against Human Dignity, Merseyside Maritime Museum, septembre, 1994, pp. 122-126.

- Small Stephen, Police and People in London. A Group of Young Black People, Londres, Policy Studies Institute, 1983.

- Tibbles Tony (dir.), Transatlantic Slavery. Against Human Dignity, Merseyside Maritime Museum, septembre, 1994.

- Trouillot Michel-Rolph, Silencing the Past : Power and the Production of History, Boston, Beacon Press, 1995.

- Tulloch Carol, "Picture this : the 'Black' curator", in Littler Jo et Naidoo Roshi (dir.), The Politics of Heritage. The Legacies of "Race", Londres et New York, Routledge, 2005.

- Visram Rozina, Asians in Britain. 400 Years of History, Londres, Pluto Press, 2002.

- Wallace Elizabeth Kowaleski, The British Sale Trade \& Public Memory, New York, Columbia University Press, 2006.

- Young Lola, "Rethinking heritage : cultural policy and inclusion", in Sandell Richard (dir), Museums, Society, Inequality, New York et Londres, Routledge, 2002, pp. 203-212. 


\section{Notes}

1. Par "Noir", je désigne les gens d'origine africaine et afro-caribéenne, et les gens issus de ces groupes qui s'identifient comme "métis". Eliot Joseph Benn Rose et al, Colour and Citizenship : A Report on British Race Relations, Londres, Oxford University Press for Institute of Race Relations, 1969.

2. Elizabeth Kowaleski Wallace, The British Sale Trade \& Public Memory, New York, Columbia University Press, 2006 ; Jo Littler et Roshi Naidoo (dir.), The Politics of Heritage. The Legacies of "Race", Londres et New York, Routledge, 2005.

3. Tony Tibbles (dir.), Transatlantic Slavery. Against Human Dignity, Merseyside Maritime Museum, septembre, 1994 ; Rozina Visram, Asians in Britain. 400 Years of History, Londres, Pluto Press, 2002 ; Lola Young, "Rethinking heritage : cultural policy and inclusion", in Sandell Richard (dir), Museums, Society, Inequality, New York et Londres, Routledge, 2002, pp. 203-212 ; Carol Tulloch, "Picture this : the 'Black' curator", in Jo Littler et Roshi Naidoo (dir.), The Politics of Heritage. The Legacies of "Race", Londres et New York, Routledge, 2005.

4. Brennan Ferne, "Are reparations for slavery justified?", in Human Rights Global Focus Group, Inde, International Human rights Foundation, vol. 2, n 1, mars 2005, pp. 5-17 ; Brennan Ferne, "Race, rights reparations : exploring a reparation framework for addressing trade inequality", in Hamline Journal of Public Law and Policy, vol $30, n^{\circ} 1$, automne 2008 .

5. Greenhill Eilean Hooper (dir.), Museums and Multiculturalism in Britain, Leicester, Leicester University Press, 1997.

6. Michel-Rolph Trouillot, Silencing the Past : Power and the Production of History, Boston, Beacon Press, 1995; Jo Littler et Roshi Naidoo (dir.), The Politics of Heritage. The Legacies of "Race", op. cit.

7. Peter Fryer, Staying Power : The History of Black People in Britain, Londres, Pluto Press, 1984 ; Kwame Nimako et Stephen Small, "The unfinished business of emancipation : the legacies of anglophone and dutch abolition", article présenté à l'Unfinished Emancipation and Black European Thinkers Symposium, National Institute for the Study of Dutch Slavery and its Legacy, Amsterdam, 29-30 juin 2009.

8. Stephen Small, "Contextualizing the Black presence in British Museums : representations, resources and response," in Greenhill Eileen Hooper (dir), Museums and Multiculturalism in Britain, Leicester, Leicester University Press, 1997,pp. 50-66.

9. Peter Fryer, Staying Power : The History of Black People in Britain, op. cit.

10. Robert Miles et Annie Phizacklea, White Man's Country : Racism in British Politics, Londres, Pluto Press, 1984.

11. Rozina Visram, Asians in Britain. 400 Years of History, Londres, Pluto Press, 2002.

12. Jennifer L. Morgan, Laboring Women : Reproduction and Gender in New World Slavery, Philadelphie, University of Pennsylvania Press, 2004.

13. Robert Miles et Annie Phizacklea, White Man's Country : Racism in British Politics, op. cit.

14. Beverley Bryan, Stella Dadzie et Suzanne Scafe, The Heart of the Race. Black Women's Lives in Britain, Londres, Virago, 1985.

15. Ambalavaner Sivanandan, Communities of Resistance. Writings on Black Struggles for Socialism, Londres et New York, Verso, 1990 ; Stephen Small, Racialised Barriers : The Black Experience in the United States and England, New York et Londres, Routledge, 1994.

16. Horace Campbell, Rasta and Resistance. From Marcus Garvey to Walter Rodney, Londres, A Hansib Publication, 1985.

17. Stephen Small, Police and People in London. A Group of Young Black People, Londres, Policy Studies Institute, 1983.

18. Mooira G. Simpson, Making Representations: Museums in the Post-Colonial Era, Londres et New York, Routledge, 2001 (1996).

19. Annie E. Coombes, Reinventing Africa. Museums, Material Culture and Popular Imagination in Late Victorian and Edwardian England, New Haven et Londres, Yale University Press, 1994.

20. Ce souverain, l'oba, personnage qui cumulait des fonctions religieuses et temporelles, était absolument invisible pour des raisons cultuelles. Le consul voulut passer outre, il fut massacré.

21. Lord Gifford, Wally Brown et Ruth Bundy, Loosen the Shackles. First Report of the Liverpool 8 Inquiry into Race Relations in Liverpool, Londres, Karia Press, 1989.

22. Stephen Small, "The general legacy of the atlantic slave trade", in Tibbles Tony (dir.), Transatlantic Slavery. Against Human Dignity, Merseyside Maritime Museum, septembre, 1994, pp. 122-126.

23. Jennifer L. Eichstedt et Stephen Small, Representations of Slavery. Race, Ideology and Southern Plantations Museums, Washington DC, Smithsonian Institution Press, 2002.

24. Darlene Clark Hine, Trica Danielle Keaton et Stephen Small, Black Europe and the African Diaspora, Urbana Champagne, University of Illinois Press, 2009.

25. Kwame Nimako et Stephen Small, "The unfinished business of emancipation : the legacies of anglophone and dutch abolition", op. cit. 